Goldschmidt 2021 Abstract

https://doi.org/10.7185/gold2021.5460

\section{Planetary Ice-Ocean Interfaces: Structure, Dynamics, and Astrobiological Implications}

\section{JACOB BUFFO $^{1}$, COLIN MEYER ${ }^{1}$ AND JAMIE PARKINSON $^{2}$}

\author{
${ }^{1}$ Dartmouth College \\ ${ }^{2}$ University of Oxford \\ Presenting Author: jacob.j.buffo@dartmouth.edu
}

Ocean worlds have been identified as high priority astrobiology targets due to the link between life and liquid water. Furthermore, youthful surface terrain on many icy bodies indicates they support active geophysical cycles that may facilitate ocean-ice shell-surface transport and/or near surface water reservoirs that could provide observables for upcoming missions (e.g. Europa Clipper).

Accurately interpreting spacecraft observations requires constraining the relationship between empirical characteristics of the ice shells of icy worlds and their interior dynamics. Furthermore, the composition and material transport processes occurring in planetary ice shells will impact ice-ocean world geophysics and subsurface ocean habitability. On Earth, the composition, physical characteristics, and bioburden of ocean derived ices are related to their formation history and parent fluid composition. In such systems the ice-ocean interface, which exists as a multiphase permeable ice-brine slush (or mushy layer - Figure 1), plays a fundamental role in dictating the overlying ice's properties and evolution. Inclusion of the physics governing these boundaries is a novel strategy in modeling planetary iceocean systems, and thus far has been limited to 1D approaches.

Here we present results from 2D simulations of an archetypal ice-ocean world system. We track the evolution of temperature, salinity, porosity, and brine velocity within a thickening ice shell (Figure 1) enabling us to place improved constraints on a number of ice-ocean world properties, including: the composition of planetary ice shells, the thickness and hydraulic connectivity of ice-ocean interfaces, and heterogenous dynamics/structures in the interfacial mushy layer. We show that stable eutectic horizons are likely a common feature of ice-ocean worlds and that ocean composition plays an important role in governing the structure and dynamics of the interface, including the formation of gradient rich inverted chemical gardens (Figure 2). We discuss the geophysical and astrobiological implications of our results and highlight how they can be validated by and aid in the synthesis of instrument specific measurements.

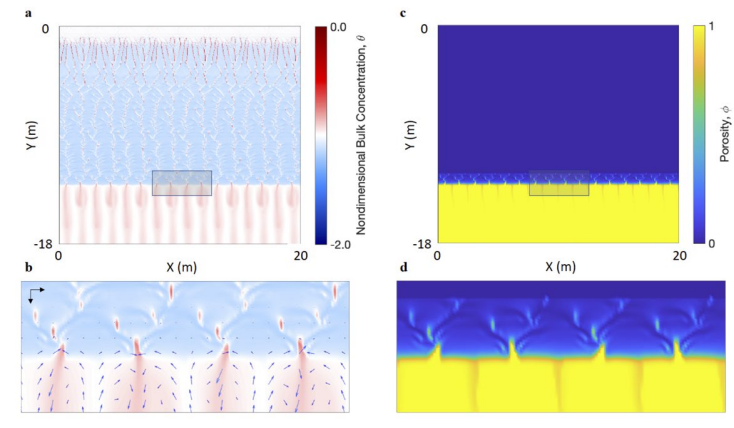

Figure 1. The compositional (bulk salinity) and structural (porosity) profile in Europa's growing ice shell. a) Two-dimensional nondimensionalized bulk salinity profile using a scale height, $H$, of $10 \mathrm{~m}$ (domain size: $18 \mathrm{~m} \times 20 \mathrm{~m})$. Where nondimensional bulk salinity, $\theta=\frac{C-C_{c}}{C_{C}-C_{i}}$. Fossilized high salinity regions can be seen in the upper ice and dense, high salinity plumes can be seen emanating from the ice-ocean interface, b) Magnified view of the shaded box in panel (a), with

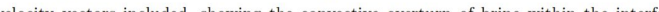
velocity vectors included, showing the convective overtun of brine within the interfacial mushy ayer. For scale, black arrows in the top left have lengths that represent Darcy velocities of $10^{-}$ $\mathrm{m} / \mathrm{s}$ c) Two-dimensional porosity profile for the same region depicted in panel (a). d) Magnified view of the shaded box in panel (c). Low porosity brine channels are associated with high salinity downwellings.

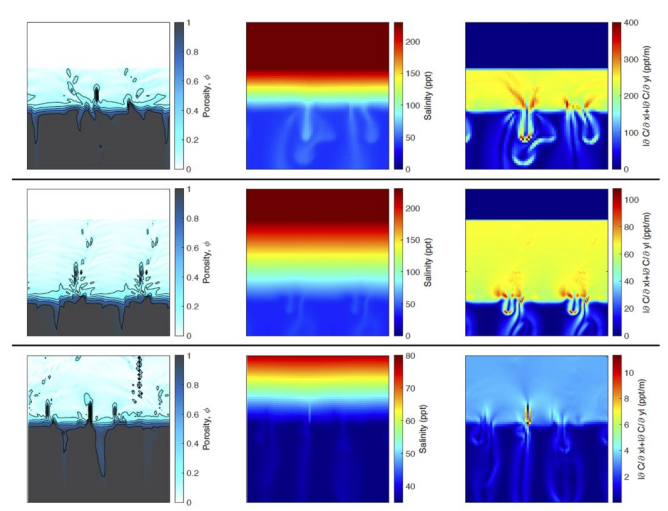

Figure 2. The physicochemical properties of brinicles on Europa. (Top-Bottom) Simulation results when the ice-ocean interface is at a depth of Top $-11 \mathrm{~m}(2.75 \mathrm{~m} \times 2.75 \mathrm{~m}$ grid), Middle $56 \mathrm{~m}(5.5 \mathrm{~m} \mathrm{X} 5.5 \mathrm{~m}$ grid), and Bottom $-1084 \mathrm{~m}$ (27.5 m x $27.5 \mathrm{~m}$ grid). (Left-Right) Porosity (contours demarcate porosities of 0.15 to 0.95 in increments of 0.2 ), brine salinity, and absolute salinity gradient $(|\partial C / \partial x|+\mid \partial C / \partial y)$ profiles during the simulations. (Note: the scale of the color bars differs between some of the images so as to highlight gradients within individual images. 